tion in insurance. Finally, the use of genetic tests exacerbates the dilemma of underwriting described above as the tests increase the amount of information available regarding a person's risk of becoming ill. Because of these distinctions between monogenic and non-genetic tests a strong case can be made for forbidding the use of tests for monogenic conditions.

Given the rapid technical advances in medical genetics, testing for a predisposition to many common disorders--such as heart disease, diabetes, and various forms of cancer-may soon be possible. For these diseases both genetic and environmental factors are usually implicated in the pathogenesis. As these diseases, like most diseases, are neither exclusively genetic nor exclusively environmental, tests for them cannot be readily distinguished from other medical tests.

The blurring of the distinction between genetic and nongenetic medical conditions does not mean that the use of tests that provide genetic information about multifactorial conditions will be no more problematic than non-genetic medical tests. Typically, genetic tests, especially those for multifactorial conditions, provide less predictive information about the course of a disease than is commonly believed. Moreover, stigmatisation and discrimination will not disappear so long as genes are believed to play a dominant part in determining our lives. The Insurance Task Force of the Human Genome Project proposed a moratorium on the use of genetic tests in health insurance until a national health care programme was in place. ${ }^{3}$ We would argue that, given the problems raised by genetic tests, a moratorium similar to that suggested by the Nuffield Council on Bioethics should be extended to all forms of insurance in which underwriting is based on medical risks. ${ }^{45}$

JOSEPH S ALPER Professor

Department of Chemistry and Center for Genetics and Public Policy,

University of Massachusetts-Boston,

Boston MA 02125,

USA

MARVIN R NATOWICZ Director

Division of Medical Genetics,

Shriver Center for Mental Retardation,

Waltham MA 02254,

USA

1 Billings PR, Kohn MA, de Cuevas M, Beckwith J, Alper JS, Natowicz MR. Discrimination as a consequence of genetic testing. Am $\mathcal{F}$ Hum Genet 1992;50:476-82.

2 US Congress, Office of Technology Assessment. Genetic counselling and cystic fibrosis carrier screening: results of a survey. Washington DC: US Government Printing Office, 1992:11-5.

3 National Institutes of Health-Department of Energy Working Group on Ethical, Legal, and Social Implications of Human Genome Research. Genetic information and health insurance. Bethesda: National Institutes of Health, 1993.

4 Nuffield Council on Bioethics. Genetic screening: ethical issues. London: Nuffield Council on Bioethics, 1993:65-74.

5 Tonks A. Ethics committee demands control on genetic screening. BMF 1993;307:1513.

\title{
Benign paroxysmal positional vertigo
}

\section{Usually self limiting}

Benign paroxysmal positional vertigo is characterised by brief but severe episodes of rotational vertigo that last less than a minute and occur on sudden changes of head position-for example, looking up or turning towards the affected ear while lying in bed.

Although the condition was first described by Bárány in $1921,{ }^{1}$ Dix and Hallpike defined the classic diagnostic test, ${ }^{2}$ which should be performed after a suggestive history has been elicited. With the patient supine, the head is lowered some $30^{\circ}$ below the level of the couch and turned some $30^{\circ}$ to $40^{\circ}$ to one side. If the test is positive the patient experiences vertigo and a rotatory nystagmus towards the undermost ear after a latent period of a few seconds. This diminishes within 50 seconds of onset-a phenomenon known as adaptation. On sitting up, the patient experiences a milder bout of vertigo, and nystagmus may occur. Any nystagmus induced by this test which does not fit this description may be positional nystagmus of central origin and warrants further neurological investigation. Occasionally, benign paroxysmal positional vertigo may occur bilaterally.

In most cases the cause is unknown and may be spontaneous degeneration within the labyrinth. ${ }^{3}$ Benign paroxysmal positional vertigo is, however, the most common vestibular disorder after head injury ${ }^{4}$ (when the symptoms and clinical course tend to be more severe) and has also been described after viral illness, ${ }^{5}$ stapes surgery, ${ }^{3}$ and chronic middle ear disease. $^{2}$

The pathological basis for the condition is believed to be displacement of the otoconia from the maculae (the sensory receptors for linear acceleration situated in the utricle and saccule) either by degeneration or by trauma. The otoconia subsequently settle on the most dependent part of the labyrinth - the cupula of the posterior semicircular canal (hence the alternative term "cupulolithiasis)." The evidence comes from two postmortem studies of known sufferers in whom basophilic deposits, thought to be macular otoconia, were found in the cupula of the posterior semicircular canal.

The displaced otoconia are believed to alter the function of the cupula by making it sensitive to linear gravitational forces. The characteristic rotatory nystagmus is due to ampullofugal stimulation of the posterior semicircular canal which causes contraction of the ipsilateral superior oblique and contralateral inferior rectus muscles. ${ }^{6}$ The latency of onset and fatiguability of the nystagmus may be explained by the delay in the onset of the ampullofugal forces and the dispersal of the otoconia in the posterior semicircular canal respectively.

Idiopathic cases tend to occur from the fifth decade onwards. ${ }^{7}$ The average recovery period is six months but, in a few patients, the symptoms persist much longer and are sufficiently troublesome to result in hospital referral. Electronystagmography may show a positional nystagmus, and caloric responses may be normal, increased, decreased, or absent, so these investigations are not routinely performed in this condition. As the condition is generally self limiting, management is expectant, based on an explanation of the condition, reassurance of its benign nature, and the prescription of labyrinthine sedatives when indicated.

The Cooksey Cawthorne exercises, described in 1945, were designed to rehabilitate patients with disabling vertigo after head injury or inner ear surgery. These consist of a series of graduated head and body exercises that aim to restore balance and confidence. ${ }^{8} \mathrm{~A}$ canalith repositioning procedure has been described by Epley; this aims to disperse the otoconia from the posterior semicircular canal into the utricle by specific, supervised, head manoeuvres. This technique, however, requires premedication, needs to be accurately performed, and may require repeated hospital visits until the symptoms 
are abolished. Success rates of $100 \%$ have been claimed. ${ }^{9}$

Recently, Brandt has developed the technique of vestibular habituation training, specifically for use in benign paroxysmal positional vertigo - it encourages patients to repeatedly adopt the position that provokes their attacks until they diminish or disappear completely. Vestibular habituation training is easily learned by the patient and repeated at home. Response rates over $90 \%$ have been claimed, ${ }^{5}$ but whether the technique disperses otoliths, encourages central compensation for the abnormal vestibular input, or increases patients' confidence in dealing with potentially frightening symptoms is unknown.

In the few patients who develop persistent and disabling symptoms surgery may be considered, but positional nystagmus of central origin should be excluded first. The operation of singular neurectomy, which selectively denervates the posterior semicircular canal, is a logical and effective procedure, producing up to $100 \%$ success rates. The operation carries a substantial risk of sensorineural deafness and is technically demanding. ${ }^{10}$ Intracranial vestibular nerve section has been performed and, in those patients with no useful hearing in the affected ear, labyrinthectomy is an option.

Recently transmastoid procedures that occlude the posterior canal with bone dust or partition the labyrinth using a laser have been described. ${ }^{112}$ These are simpler to perform and reportedly carry less risk of permanent sensorineural deafness than singular neurectomy.
Clinical and pathological evidence suggests that the symptoms of benign paroxysmal positional vertigo arise from the posterior semicircular canal. Further pathological studies are required, however, before the theory of cupulolithiasis can be confidently supported. The newly described technique of vestibular habituation training is likely to benefit most patients with the condition. The few who have persistent and disabling symptoms should be considered for occlusion of their posterior semicircular canal.

STUART W DENHOLM Senior registrar in ENT

Singleton Hospital,

Swansea, SA2 8AQ 1 Bárány R. Diagnose von Krankheitserscheinungen im Bereiche des Otolithenapparates. Acta
Otorlaryngol 1921;2:434-7.
2 Dix MR, Hallpike CS. The pathology, symptomatology and diagnosis of certain disorders of the
vestibular system. Proc Roy Soc Med 1952;45:341-54.
3 Schuknecht HF. Cupulolithiasis. Arch Otolaryngol 1969;90:765-78.
4 Barber HO. Positional nystagmus, especially after head injury. Laryngoscope 1964:74:891-944.
5 Brandt T. Man in motion. Brain 1991;114:2159-74.
6 Harbert F. Benign paroxysmal positional nystagmus. Arch Ophthalmol 1970;84:298-302.
7 Baloh RW, Honrubia V, Jacobson K. Benign postural vertigo, clinical and oculographic features in
240 cases. Neurology 1987;37:371-8.
8 Cooksey FS. Rehabilitation in vestibular injuries. Proc Roy Soc Med 1945;39:273-8.
9 Epley JM. The canalith repositioning procedure: for treatment of benign paroxysmal positional
vertigo. Otolaryngol Head Neck Surg 1992;107:399-404.
10 Gacek R. Transection of the posterior ampullary nerve for relief of BPPV. Ann Otol Rhinol Laryngol
1974;83:596-605.
11 Parnes LS, McClure JA. Posterior semi circular canal occlusion in the normal hearing ear.
Otolaryngol Head Neck Surg 1991;104:52-7.
12 Anthony DF. Partitioning of the labyrinth. Application benign paroxysmal positional vertigo.
Am f Otol 1991;12:388-93.

\section{Sunbeds and the pursuit of the year round tan}

\section{Should be discouraged}

The perception of a suntan as an essential component of a socially desirable appearance is a relatively recent phenomenon. Victorian ladies preferred to remain pale and interesting, lest they be confused with the lower classes who toiled in the fields. But over the past 20 years developing and maintaining a year round suntan has become a social necessity for some people. Manufacturers of sunbeds and those promoting their use obviously have an economic interest in persuading people that a glowing tan is a visible sign of good health, and they would no doubt claim that they are merely satisfying public demand.

Some of the current promotional literature is clearly designed to reassure prospective purchasers that acquiring a tan on a sunbed is entirely safe. One large European manufacturer of sunbeds that emit ultraviolet $\mathrm{A}$ radiation states that "incorrect and uninformed reports on the negative effects of the sun and sunbeds fuel hysteria and even panic." The same manufacturer states testimonial support to suggest that using sunbeds can inhibit the development of both melanoma skin cancer and internal cancer, stimulate the immune system, and "regenerate calcium for building our bones."

Undoubtedly, this and similar sale pitches have impressed the public: an estimated 80000 machines for home use were sold in 1988 compared with 5000 in $1982 .{ }^{1}$ This is in addition to the widely available tanning facilities in beauty salons and municipal and private sports centres. Regular users tend to be young (16-30 years), female, ${ }^{2}$ and given the cost, relatively affluent. When suntanning facilities are located at sites where sport and fitness are promoted (for example, swimming pools and gymnasiums) there is probably an implicit assumption of "health by association." Little evidence exists that recent publicity campaigns alerting people to the dangers of excessive exposure to ultraviolet light have so far had much effect.
Almost all modern commercially available sunbeds emit predominantly ultraviolet A radiation (315-380 nm), although small amounts of ultraviolet $B$ radiation (280-315 nm) are also present. The degree of tanning produced by these machines varies and depends largely on the skin type of the user. ${ }^{3}$ From the purely cosmetic aspect, patients who burn or tan poorly in natural sunlight will fare no better on a sunbed, although the risks of producing acute erythema (sunburn) may be somewhat reduced. Even subjects who develop some degree of tanning will find that this offers only very limited protection against subsequent burning when exposed to natural sunlight. ${ }^{3}$ This is clearly important to those who believe that a few sessions on a sunbed before a fortnight on a Mediterranean beach will prevent sunburn, especially as acute blistering sunburn is now considered to be a major risk factor for the subsequent development of malignant melanoma. ${ }^{4}$

Repeated exposure to ultraviolet A radiation often leads to increased skin wrinkling, irregular pigmentation, and altered skin texture (photoaging). These are not usually considered to be cosmetically desirable effects, and some people subsequently seek reversal of these changes (at even greater expense). Equally undesirable are the deeply pigmented freckles ("sunbed lentigines") that occur in some users, especially as dysplastic cellular changes may occur within these lesions. ${ }^{5}$ Regular users may also develop increased skin fragility and blistering (pseudoporphyria). ${ }^{6}$

Some people should be specifically warned against the use of sunbeds. These include patients with a known pre-existing photodermatosis such as lupus erythematosus and those taking drugs or using cosmetics with a photosensitising potential. Patients with multiple melanocytic naevi, especially those with the dysplastic naevus syndrome, should also avoid sunbeds (as well as undue exposure to natural sunlight). 